Fanum

Sociológico

\section{Forum Sociológico}

Série II

$29 \mid 2016$

Número 29

\title{
A gestão da performance nas culturas juvenis
}

Performance management in youth cultures

\section{Elsa Pegado}

\section{OpenEdition}

\section{Journals}

Edição electrónica

URL: https://journals.openedition.org/sociologico/1481

DOI: $10.4000 /$ sociologico. 1481

ISSN: 2182-7427

\section{Editora}

CICS.NOVA - Centro Interdisciplinar de Ciências Sociais da Universidade Nova de Lisboa

Refêrencia eletrónica

Elsa Pegado, «A gestão da performance nas culturas juvenis», Forum Sociológico [Online], 29 | 2016, posto online no dia 31 dezembro 2016, consultado o 31 março 2022. URL: http://

journals.openedition.org/sociologico/1481; DOI: https://doi.org/10.4000/sociologico.1481

(c) CICS.NOVA 


\title{
A GESTÃO DA PERFORMANCE NAS CULTURAS JUVENIS PERFORMANCE MANAGEMENT IN YOUTH CULTURES
}

\author{
Elsa Pegado \\ Instituto Universitário de Lisboa (ISCTE-IUL), Centro de Investigação e Estudos de Sociologia (CIES-IUL) \& Egas Moniz, \\ Centro de Investigação Interdisciplinar (CiiEM)
}

\begin{abstract}
Resumo
Este artigo procura contribuir para uma reflexão sobre o lugar que a gestão da performance ocupa nas culturas juvenis, focando em particular as percepções dos jovens sobre os consumos de performance, entendidos como o consumo de medicamentos e/ou produtos terapêuticos com finalidades de gestão do desempenho neuro/cognitivo e/ou físico/corporal. A análise tem como base material empírico resultante da componente extensiva de um projecto de pesquisa sobre consumos de performance na população jovem (18-29 anos) em Portugal, designadamente um inquérito por questionário a uma amostra $(n=1483)$ que abrangeu estudantes do Ensino Superior e trabalhadores sem formação superior. Os resultados revelam uma relativa permeabilidade nas culturas juvenis à ideia de gestão da performance, embora essa permeabilidade não seja homogénea e assuma modulações, quer em função de diferentes critérios de legitimação dos consumos, quer em termos da diversidade social e dos contextos culturais em que os jovens se situam.
\end{abstract}

Palavras-chave: culturas juvenis, gestão da performance, consumos de performance, corpo

\begin{abstract}
This article seeks to contribute to a discussion of the place of performance management in youth cultures. For that purpose, it focuses on young people's perceptions concerning performance consumptions, defined as the consumption of medicines and/or natural therapeutic products for purposes of mental/cognitive and/or physical/body performance. The empirical data for this analysis was drawn from the extensive stage of a research project on performance consumptions among young people (aged 18-29) in Portugal, which consisted of a survey to a sample $(n=1483)$ that comprised both university students and young workers without higher education. The results reveal a certain permeability in youth cultures to the notion of managing performance. However, this permeability is not homogeneous; it is modulated according to the different criteria of legitimacy associated with performance consumptions, as well as to the social and cultural diversity of the contexts in which young people are integrated.
\end{abstract}

Keywords: youth cultures, performance management, performance consumptions, body

\section{Introdução}

A performance como ideologia (Heilbrunn, 2004) ou o culto da performance (Ehrenberg, 1991), quer no domínio neuro/cognitivo, quer no físico/ corporal, têm vindo a ser apontados como traços característicos da modernidade. Tendo começado por ser circunscrita a contextos específicos, em que a competitividade constitui o valor dominante - como é o caso do desporto de competição ou do universo empresarial -, a pressão social para a performance tem vindo a estender-se para lá destes espaços e a adquirir crescente importância nos quotidianos dos indivíduos em geral, em que as exigências para a optimização da capacidade produtiva são cada vez mais prementes (Williams e Boden, 2004).

No presente artigo, a noção de performance, significa, pois, a necessidade e a capacidade de desempenhar, de forma socialmente adequada, o conjunto de papéis sociais representados em diversas 
esferas da vida - por exemplo, na família, na escola, no trabalho -, caracterizados por diferentes imperativos de desempenho. Se esta definição nada tem de novo, o que suscita novas reflexões é o facto de, nas sociedades modernas, a possibilidade de gerir essa performance ter vindo a ser crescentemente alimentada por um florescente mercado de oferta de produtos e serviços orientados para (a criação de) essas necessidades, no quadro da cultura de consumo (Featherstone, 1982). Uma das formas em que essa gestão se pode traduzir passa pelo recurso aos consumos de performance, aqui entendidos como o consumo de medicamentos e/ou produtos terapêuticos naturais com finalidades de gestão do desempenho neuro/cognitivo e/ou físico/corporal (Lopes et al., 2015; Lopes e Rodrigues, 2015).

Estes consumos têm vindo a ser objecto de pesquisas empíricas que se debruçam especificamente sobre a população jovem, quer incidindo no desempenho cognitivo em contexto académico, quer focando o desempenho físico/corporal, quer ainda abarcando o desempenho nos vários domínios e abrangendo jovens de diferentes perfis sociais, com vivências inscritas em diferentes contextos (Lopes et al., 2005; Lopes e Rodrigues, 2015), no pressuposto de que a população jovem constitui um enfoque empírico particularmente profícuo para a exploração de novas formas de gestão da performance. Tal não significa tomar a juventude como uma categoria socialmente homogénea, mas sim assumi-la como "socialmente dividida, em função dos seus interesses, das suas origens sociais, das suas perspectivas e aspirações" (Pais, 1990: 149). É neste sentido que ganha relevância mobilizar o conceito de culturas juvenis, que retomaremos mais à frente.

O presente artigo procura contribuir para uma reflexão sobre o lugar que a gestão da performance ocupa nas culturas juvenis, a partir de dois focos analíticos. O primeiro corresponde à discussão das percepções dos jovens inseridos em diferentes contextos da vida quotidiana - designadamente académicos e profissionais - sobre a gestão da performance. O segundo remete para a exploração das suas percepções relativamente àquela gestão através do recurso a medicamentos e/ou produtos naturais.

A análise é empiricamente sustentada nos resultados de um estudo, de âmbito nacional, sobre consumos de performance na população jovem em Portugal (18-29 anos), abrangendo jovens estudantes do Ensino Superior e jovens trabalhadores sem formação superior ${ }^{1}$. Importa deixar claro que a análise aqui desenvolvida não foca a dimensão das práticas sociais de consumo, já abordadas noutras publicações resultantes desse estudo (cf. Clamote, 2015; Lopes et al., 2015; Lopes e Rodrigues, 2015; Raposo, 2016), mas sim as atitudes face à perfor- mance e face à possibilidade de a gerir através de consumos medicamentosos, sejam fármacos ou naturais. Procura-se, assim, responder às seguintes questões: em que medida a população jovem revela alguma permeabilidade à ideia de gestão da performance e que diferenciações se verificam consoante os diferentes contextos sociais juvenis? Quais as percepções sobre a legitimidade dos consumos de performance que emergem nos discursos dos jovens e que critérios são mobilizados para as diferentes atribuições de legitimidade?

\section{Culturas juvenis e performance}

O conceito de culturas juvenis, entendidas enquanto "conjuntos de crenças, valores, símbolos, normas e práticas que determinados jovens dão mostras de compartilhar" (Pais, 1990: 140), é mobilizado, no presente artigo, para dar conta da diversidade contextual que molda as vivências quotidianas dos jovens. Como propõe Machado Pais (1990), a juventude pode ser olhada em torno de dois eixos semânticos: como aparente unidade (quando referida a uma fase de vida) e como diversidade (quando estão em jogo diferentes atributos sociais que fazem distinguir os jovens uns dos outros). Várias pesquisas têm demonstrado como esses atributos sociais constituem poderosos diferenciadores das vivências da juventude como fase da vida (cf. Shildrick e MacDonald, 2006), não só em termos de desiguais trajectórias escolares e profissionais - mais ou menos qualificadas -, mas também de acesso a experiências em contextos quotidianos de vida, onde se vão construindo culturas específicas (por exemplo culturas académicas e culturas laborais). Na sociedade portuguesa em particular, em que, não obstante um significativo aumento da escolaridade da população, a percentagem de jovens que completou o Ensino Superior continua a ser reduzida - designadamente por comparação à média europeia ${ }^{2}$-, a qualificação académica constitui um importante atributo diferenciador.

A abordagem às culturas juvenis aqui privilegiada não se foca, como parte das pesquisas sobre culturas juvenis, na juventude espectacular, exuberante (frequentemente a partir das suas manifestações visuais ou musicais), mas sim na juventude normal (ordinary) (Shildrick e MacDonald, 2006). Pressupõe perspectivar os jovens a partir dos seus contextos vivenciais, quotidianos (Pais, 2000). É nesses contextos, socialmente diferenciados, que os traços dessas culturas se manifestam. No presente artigo, a exploração desses contextos justifica-se na medida em que as suas características podem revelar exigências de performance particulares, que se constituem enquanto pressões (ou mesmo imperativos) para investimentos direccionados para a gestão da performance. Esta dimensão analítica 
traduz-se na opção metodológica de abranger jovens que se encontrem a frequentar o Ensino Superior e jovens inseridos no mercado de trabalho, sem frequência desse nível de ensino. Efectivamente, é expectável que os imperativos de desempenho quotidiano sejam distintos no universo académico e no universo laboral, gerando percepções diferenciadas face à performance.

Para lá desta diversidade social, que é analiticamente explorada através da relação entre as atitudes face à gestão da performance e o género e o perfil de actividade da população jovem - estudantes e trabalhadores -, importa considerar os jovens na sua unidade geracional, a juventude como uma fase da vida. Um dos traços que tem vindo a ser salientado é o facto de os jovens investirem muito no seu quotidiano; entre os jovens, o tempo presente tem ascendente sobre o passado e sobre o futuro (Pais, 1990). Nesta linha, outras propostas têm enfatizado o facto de as culturas juvenis contemporâneas partilharem valores que remetem para o hedonismo, o experimentalismo e o presentismo (Ferreira, 2011). O significado destas últimas noções - o experimentalismo como a tentativa de desafiar os limites, mesmo que tal implique riscos, e o presentismo como forma imediata de viver o momento presente - parece autorizar a sua mobilização para a análise das percepções e atitudes face à gestão da performance e, em particular, face aos consumos de performance. São, efectivamente, atributos que encontrarão eco nas expectativas de resultados com o consumo de medicamentos ou outros produtos para a melhoria do desempenho físico ou cognitivo: resultados rápidos, sem empreender os esforços que caracterizam investimentos orientados por lógicas de ascetismo (Pegado, 2010). Várias pesquisas sociológicas sobre a juventude têm também associado esta fase da vida a uma maior predisposição para a experimentação e a uma correlativa maior familiaridade com experiências de risco que, em alguns casos, são simbolicamente investidas de manifestações de não conformidade social (Moura Ferreira, 2003), sem que tal signifique, no entanto, assumir como característica distintiva da juventude o seu "natural" alheamento face aos riscos.

Uma segunda dimensão analiticamente relevante corresponde às culturas corporais. Embora "os limites para a aferição sociológica da 'juventude' não sejam de natureza eminentemente biológica e não se determinem exclusivamente pela idade dos indivíduos, certo é que, socialmente, ser jovem passa também pela codificação etária de um dado modelo de corporeidade (...)" (Ferreira, 2011: 258). Tal significa que ser jovem é ser portador de uma condição corporal, consubstanciada quer numa determinada imagem, quer em determinadas capacidades de desempenho físico, normativamente definidas para um grupo etário relativamente deli- mitado. Nas sociedades contemporâneas, o "corpo jovem" assume-se como o modelo idealizado de corporeidade, amplamente veiculado através dos meios de comunicação social e, em particular, da publicidade, passível de ser atingido através de investimentos de natureza vária (onde se incluem os investimentos medicamentosos), crescentemente mercantilizados, que a indústria da corporalidade, no quadro da cultura de consumo (Featherstone, 1982), vem proporcionar. Esta imagem do "corpo jovem" terá consequências nas avaliações e regimes de vigilância e/ou modificação corporal que os próprios jovens fazem e impõem aos seus corpos. Pode traduzir-se, pois, em investimentos corporais que integram a categoria de corpo projecto, a partir da acepção de Shilling (1993), ancorados em valores de hedonismo corporal e orientados para a performatividade física e/ou estética.

É neste quadro que importa analisar as percepções dos jovens sobre a gestão da performance e sobre os investimentos que podem ser mobilizados para o fazer, designadamente os consumos de performance. Estes consumos tanto podem visar uma lógica de recuperação de níveis considerados "normais", como uma lógica de melhoria desses níveis, ultrapassando os limites "naturais". Trata-se de investimentos que, na sua intencionalidade, são exteriores a objectivos terapêuticos (de manutenção ou reposição da saúde), mas que não deixam de questionar as tradicionais fronteiras entre doença, saúde e bem-estar, bem como a distinção conceptual entre consumos terapêuticos e consumos de melhoria (Coveney, Gabe e Williams, 2011). Efectivamente, se estas categorias visam estabelecer a fronteira entre o que está dentro e fora do campo da saúde, um dos seus limite analíticos em matéria de consumos de performance é o facto de que "o espetro de finalidades de melhoria não se esgota na procura de otimização das capacidades de desempenho, já que, não raro, o objetivo não é superar o normal, mas tão-somente alcançá-lo de forma mais rápida ou com menor esforço" (Lopes e Rodrigues, 2015: 14).

\section{Metodologia}

A análise e discussão que a seguir se desenvolvem toma como material empírico os resultados da componente extensiva de um projecto de pesquisa sobre consumos de performance ${ }^{3}$ na população jovem em Portugal, onde se incluem fármacos e produtos naturais, na continuidade de outras pesquisas que vêm revelando a crescente expressão do natural nos consumos terapêuticos (Lopes, 2010; Lopes et al., 2012). Em particular, neste artigo procede-se à análise de alguns indicadores incluídos num inquérito por questionário aplicado a uma amostra de jovens residentes em Portugal continental 
$(n=1483)$ - amostra por quotas não proporcional -, com idades compreendidas entre os 18 e os 29 anos, uma parte dos quais a frequentar o Ensino Superior $(70 \%)^{4}$ e outra parte sem frequência universitária, integrada no mercado de trabalho (30\%). Estes últimos incluem trabalhadores de atendimento ao público em dois tipos de empresas: call-centers (51\%) e mega-stores (49\%). Os jovens do Ensino Superior abrangem estudantes de diferentes áreas científicas: Saúde $(52,7 \%)$, Ciências Sociais $(18,3 \%)$, Engenharia (19,3\%) e Artes (9,7\%). Na distribuição por sexo, as mulheres representam $59,2 \%$ e os homens $40,8 \%$.

Não sendo uma amostra estatisticamente representativa, a construção da amostra obedeceu a critérios de representatividade teórica (Ferreira, 1986). Os estudantes estão intencionalmente sobrerrepresentados por duas ordens de razões: primeiro, porque a literatura internacional aponta para uma maior expressão dos consumos de performance em contextos académicos; segundo, porque se pretendia abranger uma diversidade de áreas de formação, inquirindo um número suficientemente elevado de estudantes em cada uma dessas áreas de modo a possibilitar comparações com densidade analítica adequada. A sobre-representação dos estudantes da área da Saúde, também intencional, decorre da hipótese de que estes, por via dos conteúdos da sua formação académica, estariam mais familiarizados com os consumos de performance.

A aplicação dos questionários decorreu nos estabelecimentos de Ensino Superior, em espaços próprios para o efeito, após obtidas as autorizações institucionais necessárias. Os jovens trabalhadores foram contactados nas empresas e inquiridos em locais reservados cedidos por estas. O contacto com os inquiridos foi assegurado pela equipa de investigadores, constituída por sociólogos, sem interferência dos interlocutores institucionais; no caso das empresas, para salvaguardar qualquer equívoco quanto às finalidades e anonimato das respostas, foi também distribuída uma nota informativa prévia a todos quantos se voluntariaram para participar no estudo. Os questionários, anónimos e auto-administrados, foram aplicados com a presença da equipa de investigadores, de forma a assegurar um melhor controlo metodológico sobre as condições de aplicação ${ }^{5}$. A aplicação decorreu de Setembro de 2012 a Fevereiro de 2013, após a realização de um pré-teste a uma amostra de 90 inquiridos, com uma composição sociodemográfica equivalente à amostra final. O inquérito foi precedido por uma etapa inicial de pesquisa qualitativa, constituída pela realização de grupos focais (10 sessões; 57 participantes), que garantiu uma aproximação exploratória ao universo em análise e permitiu informar as opções metodológicas que presidiram à construção dos indicadores e da estrutura do questionário, bem como afinar critérios para a construção da amostra. A par da observância dos requisitos éticos da investigação em Ciências Sociais - o anonimato dos sujeitos e das instituições e o carácter estritamente voluntário da participação - foi igualmente observado o consentimento informado para as fases da pesquisa que implicaram gravação, designadamente no caso dos grupos focais.

Do vasto conjunto de indicadores do questionário aplicado, mobilizam-se, para o presente artigo, aqueles que constituem contributos para a discussão sobre o lugar que a gestão da performance ocupa nas culturas juvenis. Essa discussão é realizada a partir de duas dimensões: uma dimensão das representações, que remete para as imagens dos jovens sobre o seu desempenho a vários níveis (cognitivo, corporal e social); e uma dimensão que remete para as percepções sobre a legitimidade dos consumos de performance, de diferentes naturezas e em diferentes contextos. O objectivo é o de proporcionar uma visão panorâmica, de carácter extensivo, bem como dar conta das principais tendências e diferenciações que as distribuições destes indicadores e o seu cruzamento com variáveis sociodemográficas sugerem. Procura-se ainda testar que relações se estabelecem entre, por um lado, auto-representações de desempenho e consumos efectivos e, por outro, entre percepções de legitimidade e esses consumos.

\section{Auto-representações sobre desempenho}

Os indicadores que remetem para as auto-representações dos jovens inquiridos sobre o seu desempenho nos vários domínios considerados revelam um padrão semelhante no que respeita à capacidade intelectual e de aprendizagem e à imagem corporal, caracterizado por uma distribuição relativamente equitativa entre os que gostariam de melhorar e os que estão satisfeitos com esses aspectos, e uma percentagem bastante reduzida dos que declaram não se preocupar (cf. Tabela 1). Tal parece ser revelador de uma relativa equivalência das valorações atribuídas pelos jovens ao físico e ao cognitivo e, simultaneamente, uma avaliação em que a melhoria se coloca como um cenário desejável para um número muito significativo de jovens (cerca de metade dos inquiridos), o que, não autorizando a assunção de predisposições para consumos de melhoria, permite, pelo menos, salientar a relevância dessa melhoria no seu horizonte representacional.

Já quanto à capacidade de relacionamento com os outros, categoria que procura operacionalizar o desempenho social, a maioria dos jovens consideram-se satisfeitos e pouco menos de um quarto indica que gostaria de melhorar este aspecto.

A análise da distribuição sociodemográfica destes indicadores revela algumas diferenças merecedoras de destaque ${ }^{6}$. A primeira respeita à 
Tabela $1 \triangleright$ Auto-representações sobre desempenho social, cognitivo e imagem corporal

\begin{tabular}{|c|c|c|c|c|c|c|c|}
\hline \multirow{2}{*}{$\begin{array}{l}\text { Auto-representações sobre } \\
\text { desempenho }\end{array}$} & \multicolumn{2}{|c|}{ Gostaria de melhorar } & \multicolumn{2}{|c|}{ Está satisfeito } & \multicolumn{2}{|c|}{ Não se preocupa } & \multirow{2}{*}{ Total } \\
\hline & n & $\%$ & $n$ & $\%$ & $\mathbf{n}$ & $\%$ & \\
\hline $\begin{array}{l}\text { A sua capacidade intelectual } \\
\text { e de aprendizagem }\end{array}$ & 726 & 49,2 & 726 & 49,2 & 23 & 1,6 & $\begin{array}{c}100 \% \\
(n=1475)\end{array}$ \\
\hline A sua imagem corporal & 688 & 46,7 & 717 & 48,6 & 69 & 4,7 & $\begin{array}{c}100 \% \\
(n=1474)\end{array}$ \\
\hline $\begin{array}{l}\text { A sua capacidade de } \\
\text { relacionamento com os } \\
\text { outros }\end{array}$ & 337 & 22,9 & 1059 & 71,9 & 76 & 5,2 & $\begin{array}{c}100 \% \\
(n=1472)\end{array}$ \\
\hline
\end{tabular}

segmentação de género que se verifica, traduzida num maior desejo declarado de melhorar os vários aspectos por parte da população feminina (respetivamente, $54,3 \%, 48,2 \%$ e $25,1 \%$, enquanto as percentagens correspondentes na população masculina são de $41,9 \%$, 44,4\% e 19,7\%). Por sua vez, verifica-se uma manifestação de indiferença ("não se preocupa") mais expressiva por parte dos jovens do sexo masculino $(P<0,001 ; P=0,018 ; P=0,016)$.

Esta segmentação é igualmente visível quando se considera o perfil de actividade dos jovens estudantes do Ensino Superior ou trabalhadores. As auto-representações sobre o desempenho cognitivo e sobre a imagem corporal revelam padrões contrastantes nos perfis de actividade, com a população estudantil a manifestar menos satisfação com a capacidade intelectual e de aprendizagem (45,9\%) e a declarar o desejo de melhoria $(52,6 \%)$, enquanto na população laboral a distribuição é invertida (56,9\% e $41,3 \%$, respectivamente) $(P<0,001)$. Os níveis de satisfação com o desempenho cognitivo parecem, pois, revelar um forte enraizamento no contexto em que os jovens se movem, em que a frequência do Ensino Superior se constitui como um espaço e um tempo com exigências acrescidas a este nível. Quanto à imagem corporal, a relação também é estatisticamente significativa $(P=0,008)$, com os jovens trabalhadores a declarar menores níveis de satisfação ( $51,6 \%$ gostariam de melhorar, por relação a 44,6\% dos estudantes).

A distribuição destes indicadores tendo em conta os consumos de performance efectivos ${ }^{7}$ mostra que existe uma relação significativa $(P<0,001)$ entre as práticas de consumo (ou a sua ausência) e as auto-representações de desempenho. Tal relação traduz-se em dois tipos de associações. Por um lado, a que se verifica entre os inquiridos que manifestam o desejo de melhorar a capacidade intelectual e de aprendizagem e o consumo efectivo de produtos com essas finalidades: $70,6 \%$ dos jovens que manifestam este desejo referem consumir, enquanto a percentagem homóloga para os que declaram estar satisfeitos é de 57,1\%. Por outro lado, há igualmente uma associação entre os que gostariam de melhorar a sua imagem corporal e os que consomem produtos direccionados para o desempenho físico/corporal. Dos que manifestam esse desejo, 36,3\% consomem algum tipo de produto com essa finalidade, ao passo que entre os que se declaram satisfeitos ou não se preocupar as percentagens são de $24,3 \%$ e $23,1 \%$.

\section{Percepções sobre consumos de performance}

As percepções dos jovens sobre a legitimidade dos consumos com objectivos de gestão do desempenho neuro/cognitivo e/ou físico/corporal foram operacionalizadas, no questionário, de duas formas. Por um lado, procurando obter informação sobre as atitudes de aprovação/reprovação destes consumos de forma indirecta (as percepções sobre as reacções dos outros aos hipotéticos consumos do próprio), estabelecendo assim uma hierarquia do que é aceitável/reprovável em torno dos medicamentos/produtos em si e das suas finalidades. Por outro lado, procurando captar essas percepções de forma directa (as opiniões do próprio sobre os consumos) em diferentes circunstâncias e contextos, e no quadro de diferentes finalidades de consumo.

Quanto ao primeiro aspecto $^{8}$, impõem-se as seguintes considerações gerais: i) existe uma clara diferenciação das percepções sobre aprovação/ /reprovação dos vários medicamentos/produtos considerados (cf. Tabela 2); ii) as percentagens dos que referem reprovação são quase sempre superiores às de aprovação; as reacções de indiferença têm, em geral, percentagens relativamente elevadas face às positivas ou negativas. Evidencia-se, pois, um posicionamento normativo tendente a uma condenação destes consumos, a que não será alheia a atribuição de risco aos medicamentos/produtos (nomeadamente farmacológicos) que já havia sido evidenciada em outras pesquisas (Raposo, 2010). Para além deste padrão geral, o que importa salientar é a relativa coincidência que se verifica entre os tipos de consumos mais reprovados e aqueles que são objecto 
Tabela $2 \triangleright$ Percepção da reacção dos amigos a consumos hipotéticos do próprio

\begin{tabular}{|c|c|c|c|c|c|c|c|c|c|}
\hline \multirow{2}{*}{ Tipo de consumos } & \multicolumn{2}{|c|}{ Aprovação } & \multicolumn{2}{|c|}{ Indiferença } & \multicolumn{2}{|c|}{ Reprovação } & \multicolumn{2}{|c|}{ Não sabe } & \multirow{2}{*}{ Total } \\
\hline & $\mathbf{n}$ & $\%$ & $\mathbf{N}$ & $\%$ & $\mathbf{n}$ & $\%$ & $\mathbf{n}$ & $\%$ & \\
\hline Bebidas energéticas & 380 & 25,9 & 906 & 61,6 & 100 & 6,8 & 84 & 5,7 & $\begin{array}{c}100 \% \\
(n=1470)\end{array}$ \\
\hline $\begin{array}{l}\text { Medicamentos para } \\
\text { melhorar a concentração/ } \\
\text { memória }\end{array}$ & 569 & 38,6 & 640 & 43,4 & 115 & 7,8 & 152 & 10,3 & $\begin{array}{c}100 \% \\
(n=1476)\end{array}$ \\
\hline Medicamentos para dormir & 225 & 15,3 & 651 & 44,3 & 358 & 24,3 & 237 & 16,1 & $\begin{array}{c}100 \% \\
(n=1471)\end{array}$ \\
\hline $\begin{array}{l}\text { Medicamentos para diminuir } \\
\text { a ansiedade em situações } \\
\text { pontuais }\end{array}$ & 543 & 36,8 & 527 & 35,8 & 193 & 13,1 & 211 & 14,3 & $\begin{array}{c}100 \% \\
(n=1474)\end{array}$ \\
\hline $\begin{array}{l}\text { Batidos de proteínas para a } \\
\text { massa muscular }\end{array}$ & 261 & 17,7 & 512 & 34,8 & 510 & 34,6 & 189 & 12,8 & $\begin{array}{c}100 \% \\
(n=1472)\end{array}$ \\
\hline $\begin{array}{l}\text { Medicamentos para } \\
\text { aumentar a resistência } \\
\text { física na prática desportiva }\end{array}$ & 172 & 11,7 & 449 & 30,5 & 637 & 43,3 & 212 & 14,4 & $\begin{array}{c}100 \% \\
(n=1470)\end{array}$ \\
\hline $\begin{array}{l}\text { Esteroides anabolizantes } \\
\text { para a massa muscular }\end{array}$ & 26 & 1,8 & 147 & 10,0 & 1152 & 78,3 & 146 & 9,9 & $\begin{array}{c}100 \% \\
(n=1471)\end{array}$ \\
\hline $\begin{array}{l}\text { Medicamentos para perder } \\
\text { peso/emagrecer/combater } \\
\text { a celulite }\end{array}$ & 134 & 9,1 & 400 & 27,2 & 770 & 52,4 & 166 & 11,3 & $\begin{array}{c}100 \% \\
(n=1470)\end{array}$ \\
\hline Haxixe ou marijuana & 90 & 6,1 & 261 & 17,7 & 1021 & 69,3 & 102 & 6,9 & $\begin{array}{c}100 \% \\
(n=1474)\end{array}$ \\
\hline $\begin{array}{l}\text { Medicamentos para se } \\
\text { manter acordado/a }\end{array}$ & 75 & 5,1 & 352 & 23,9 & 842 & 57,2 & 203 & 13,8 & $\begin{array}{c}100 \% \\
(n=1472)\end{array}$ \\
\hline
\end{tabular}

de atribuições de risco mais elevado, autorizando a hipótese de o risco estar a ser mobilizado como um dos critérios de (des)legitimação dos consumos de performance.

Na hierarquia de aprovação/reprovação que se estabelece entre os diferentes medicamentos/ /produtos e/ou finalidades, podem ser identificados três grupos de medicamentos/produtos. O primeiro integra aqueles cujos consumos obtêm percentagens de reprovação mais elevadas, acima dos $40 \%$. Os esteróides anabolizantes encabeçam esta lista (78,3\% consideram que os amigos reprovariam), seguidos pelo haxixe ou marijuana $(69,3 \%)^{9}$. De referir que, quer num quer no outro caso, poderão estar aqui a ser mobilizados critérios associados à ilicitude e/ou à transgressão conotada com o seu consumo. Fazem ainda parte deste grupo: os medicamentos para se manter acordado $(57,2 \%)$, muito acima da reprovação dos medicamentos para dormir (24,3\%), que poderão estar a ser considerados como uma resposta terapêutica a problemas de perturbação do sono e não associados a objectivos de melhoria; os medicamentos para perder peso/emagrecer/combater a celulite $(52,4 \%)$; e os medicamentos para aumentar a resistência física na prática desportiva (43,3\%). Esta ordenação indica uma segmentação das finalidades, traduzida em taxas geralmente mais elevadas de reprovação dos consumos associados a finalidades de desempenho físico/corporal do que de desempenho neuro/cognitivo.

O segundo grupo de medicamentos/produtos é constituído por aqueles cujos consumos obtêm percentagens mais elevadas de aprovação (mesmo face a um padrão de aprovação na generalidade baixo), mas em que a indiferença é ainda mais elevada ou, pelo menos, da mesma ordem de grandeza. Inclui os medicamentos para melhorar a concentração/ /memória (39\% aprovação e 43\% indiferença), os medicamentos para diminuir a ansiedade em situações pontuais ( $37 \%$ e $36 \%$ ) e as bebidas energéticas (26\% e 62\%). Este elevado peso da "aprovação" e da "indiferença" parece indiciar uma relativa banalização e desproblematização deste tipo de consumos, tendo em conta que são precisamente estes os que assumem maior expressão em termos de consumo efectivo.

O último grupo engloba os medicamentos/ /produtos em que tende a haver uma concentração na indiferença (e um pouco menos na reprovação), com percentagens baixas de aprovação, sendo constituído pelos medicamentos para dormir e pelos batidos de proteínas.

Os níveis de aprovação ou reprovação dos consumos de performance distribuem-se de forma diferenciada em função de algumas das variáveis sociodemográficas que temos vindo a invocar. 
Embora não sendo generalizável a todos os medicamentos/produtos elencados, verifica-se uma maior aprovação e menor reprovação dos medicamentos/produtos para melhorar o desempenho neuro/cognitivo (medicamentos para melhorar a concentração/memória, medicamentos para diminuir a ansiedade em situações pontuais e medicamentos para dormir) por parte das jovens do sexo feminino, por oposição aos medicamentos/produtos para melhorar o desempenho físico/corporal (batidos de proteínas para a massa muscular, medicamentos para aumentar a resistência física na prática desportiva), que reúnem níveis de aprovação superiores por parte dos jovens do sexo masculino. Exceptuam-se deste padrão os medicamentos destinados a perder peso/emagrecer, em que a percentagem de raparigas que refere reacções de aprovação é superior. Estas percepções são congruentes com os consumos efectivos, onde foi identificada a mesma tendência de associação entre investimentos neuro/ /cognitivos e raparigas (aos quais acresce o consumo de fármacos e produtos naturais para emagrecer), por um lado, e investimentos físicos/corporais e rapazes, por outro. Vão também ao encontro de uma maior disponibilidade para a melhoria do desempenho cognitivo por parte das jovens inquiridas, atrás referida. Não deixarão de estar ainda associadas a uma cultura de maior valorização e, correlativamente, de um maior investimento, nos resultados escolares/académicos por parte das raparigas, como vem sendo demonstrado em outros estudos desenvolvidos junto de estudantes do Ensino Superior (Costa e Lopes, 2008).

A relação com o perfil de actividade dos jovens inquiridos é significativa no caso dos consumos direccionados para a performance física/corporal (batidos de proteínas, medicamentos para aumentar a resistência física e medicamentos para emagrecer), traduzindo-se na sua maior aprovação por parte da população laboral, por comparação com os estudantes. Trata-se de mais um caso em que as percepções sobre os consumos são consonantes com as práticas, já que o consumo dos medicamentos/ /produtos em causa assume uma maior expressão entre os jovens trabalhadores (Lopes et al., 2015; Lopes e Rodrigues, 2015).

Um outro indicador que introduz diferenciação nas percepções sobre legitimidade dos consumos de performance é a prática desportiva. Um pouco mais de metade dos jovens inquiridos pratica uma actividade desportiva; além disso, a prática em regime federado assume uma expressão considerável entre a população jovem (11,9\%). Como outros estudos têm demonstrado (Ferreira, 2003), a prática de actividade desportiva é diferenciada em termos de género, com os jovens do sexo masculino com taxas bastante superiores. Entre estes, neste estudo, apenas $32,1 \%$ não são praticantes, enquanto essa percentagem soma $58,1 \%$ entre as jovens. No caso do perfil de actividade dos jovens não se verificam diferenças dignas de relevo, o que demonstra que a prática de actividade desportiva é transversal aos vários contextos sociais - estudantil, laboral - em que os jovens se integram. No caso dos consumos mais directamente relacionados com essa prática - os batidos de proteínas para a massa muscular e os medicamentos para aumentar a resistência física na prática desportiva - a relação entre a prática desportiva e a percepção da legitimidade dos consumos é significativa. Os praticantes federados aprovam mais e/ou reprovam menos estes consumos, relativamente aos praticantes não federados, cujas percentagens de aprovação e de reprovação estão mais próximas dos não praticantes. Trata-se de mais um dado que vem evidenciar a pertinência analítica de atender aos contextos de inserção dos jovens - e os imperativos de desempenho que os caracterizam -, como potenciadores de atitudes de aceitação ou rejeição da possibilidade de melhoria da performance e, em particular, de o fazer através do recurso a medicamentos ou produtos naturais.

A análise das percepções sobre a legitimidade dos consumos tendo em conta os consumos efectuados revela um padrão que, embora não extensível a todas as categorias de medicamentos/produtos e a todas as finalidades, tende a associar as percepções de aprovação/reprovação às experiências efectivas de consumo. Quer dizer que a maior aceitação do consumo de determinados produtos tem correspondência com um perfil de consumo orientado para as mesmas finalidades, indiciando a relação dialéctica que se estabelece entre a experiência do consumo e as percepções sobre os consumos.

Um outro olhar sobre as percepções da legitimidade dos consumos traduziu-se na análise da distribuição do posicionamento dos inquiridos face a um conjunto de asserções que ilustram diferentes tipos de situações, contextos e circunstâncias de consumo, em que emergem três padrões, que variam de acordo com critérios diferenciados, seja o da natureza dos produtos, seja o das finalidades de consumo (cf. Tabela 3).

Em primeiro lugar, verifica-se que os consumos que remetem para uma legitimação que podemos designar por contextual ou circunstancial, onde se incluem os produtos usados para finalidades circunstanciais e/ou contextuais (épocas de exames, melhoria dos resultados escolares, melhoria da produtividade no local de trabalho), tendem a ser legitimados pelos jovens inquiridos. A maioria concorda que estes consumos se justificam (A), como tal discordando do argumento da existência de situações de injustiça relativa - batota nos resultados escolares (B), ou concorrência desleal entre os colegas no local de trabalho (C). 
Tabela $3 \triangleright$ Posicionamentos face a diferentes tipos e circunstâncias de consumo

\begin{tabular}{|c|c|c|c|c|c|c|c|}
\hline \multirow{2}{*}{ Tipos e circunstâncias de consumo } & \multicolumn{2}{|c|}{ Concorda } & \multicolumn{2}{|c|}{ Discorda } & \multicolumn{2}{|c|}{ Não sabe } & \multirow{2}{*}{ Total } \\
\hline & $\mathbf{n}$ & $\%$ & $\mathbf{n}$ & $\%$ & $\mathbf{n}$ & $\%$ & \\
\hline $\begin{array}{l}\text { A. Em épocas de frequências e exames, } \\
\text { justifica-se o consumo de medicamentos } \\
\text { ou outros produtos para melhorar a } \\
\text { concentração ou a memória. }\end{array}$ & 989 & $66,7 \%$ & 468 & $31,6 \%$ & 25 & $1,7 \%$ & $\begin{array}{c}100 \% \\
(n=1482)\end{array}$ \\
\hline $\begin{array}{l}\text { B. Tomar medicamentos ou outros produtos } \\
\text { para melhorar os resultados escolares/ } \\
\text { académicos equivale a fazer batota. }\end{array}$ & 304 & $20,5 \%$ & 1134 & $76,6 \%$ & 43 & $2,9 \%$ & $\begin{array}{c}100 \% \\
(n=1481)\end{array}$ \\
\hline $\begin{array}{l}\text { C. Tomar medicamentos ou outros } \\
\text { produtos para melhorar a produtividade } \\
\text { no local de trabalho cria situações de } \\
\text { concorrência desleal entre os colegas. }\end{array}$ & 469 & $31,7 \%$ & 881 & $59,5 \%$ & 130 & $8,8 \%$ & $\begin{array}{c}100 \% \\
(n=1480)\end{array}$ \\
\hline $\begin{array}{l}\text { D. O consumo de esteroides anabolizantes } \\
\text { é aceitável em certas actividades } \\
\text { desportivas, desde que seja } \\
\text { devidamente controlado. }\end{array}$ & 272 & $18,4 \%$ & 1109 & $74,8 \%$ & 101 & $6,8 \%$ & $\begin{array}{c}100 \% \\
(n=1482)\end{array}$ \\
\hline $\begin{array}{l}\text { E. Só em casos de obesidade devem ser } \\
\text { consumidos medicamentos ou outros } \\
\text { produtos para emagrecer. }\end{array}$ & 789 & $53,3 \%$ & 627 & $42,4 \%$ & 64 & $4,3 \%$ & $\begin{array}{c}100 \% \\
(n=1480)\end{array}$ \\
\hline $\begin{array}{l}\text { F. Não é aceitável tomar medicamentos } \\
\text { para ficar mais calmo em situações que } \\
\text { provocam ansiedade, sem prescrição } \\
\text { médica. }\end{array}$ & 699 & $47,3 \%$ & 726 & $49,1 \%$ & 54 & $3,7 \%$ & $\begin{array}{c}100 \% \\
(n=1479)\end{array}$ \\
\hline
\end{tabular}

* As categorias "concorda" e "discorda" resultam de uma agregação de quatro categorias em que os inquiridos se podiam posicionar: "concorda totalmente", "concorda parcialmente", "discorda parcialmente" e "discorda totalmente".

Já no que respeita aos casos que poderíamos classificar de legitimação pericial, ou seja, em que são avançados critérios de validação do consumo por parte de profissionais de saúde (de forma mais ou menos explícita), prevalece um padrão de relativo equilíbrio entre a legitimidade e a ilegitimidade, com valores próximos nas categorias "concordo" e "discordo", revelador de uma maior ambiguidade. Integram esta categoria os produtos para emagrecer (E), em que a asserção remete para a legitimação do consumo pela existência de uma condição medicalizada e, pelo menos implicitamente, patologizada (a obesidade); os produtos para a ansiedade (F), em que essa legitimação surge por via da intervenção directa do médico, através da prescrição; e ainda os esteróides anabolizantes, em que a asserção remete para o seu uso "devidamente controlado" (D).

As percepções sobre a legitimidade do consumo de esteróides anabolizantes constituem precisamente um caso singular, pelo seu afastamento do padrão de respostas relativo à legitimação pericial. Em consonância com as respostas ao indicador anterior - o escalonamento da legitimidade -, estes produtos voltam a figurar como o caso paradigmático da ilegitimidade: $74,8 \%$ dos inquiridos discordam da aceitabilidade do seu uso. Nesta avaliação, terá porventura mais importância o tipo de produto em si do que o controlo do seu consumo.

\section{Síntese conclusiva}

O panorama traçado neste artigo corresponde a um primeiro patamar de aproximação analítica à gestão da performance no quadro das culturas juvenis. Através da análise dos indicadores incluídos no questionário que remetem para esta dimensão, foi possível explorar as auto-representações sobre o desempenho e as percepções sobre a legitimidade dos consumos de performance, e ainda analisar a relação entre essas percepções e os consumos efectivos dos inquiridos. Esta análise permitiu também demonstrar empiricamente a constatação de que estas representações e percepções são socialmente diferenciadas.

Dos resultados obtidos, importa destacar três aspectos. Em primeiro lugar, a existência de uma avaliação do próprio desempenho, quer corporal, quer cognitivo, em que o desejo de melhoria é declarado, revelando assim como a melhoria está presente no horizonte representacional dos jovens portugueses.

Em segundo lugar, a constatação de uma clara hierarquia de legitimidade entre os consumos, que parece reportar-se ora à natureza dos produtos em $\mathrm{si}$, ora às suas finalidades e aos contextos em que são consumidos. Nesta hierarquia, os consumos associados a finalidades de desempenho neuro/cognitivo são, em geral, considerados mais legítimos que os direccionados para o desempenho físico/corporal. 
Acresce que no caso de os consumos de performance serem objecto de uma validação pericial, verifica-se um padrão de relativo equilíbrio entre declarações de legitimidade e de ilegitimidade, enquanto o consumo de produtos usados para finalidades circunstanciais e/ou contextuais, ligadas aos imperativos da vida quotidiana (resultados académicos, produtividade no trabalho) tende a ser legitimado. Esta conclusão é convergente com a análise de alguns autores, segundo os quais os valores dominantes das culturas juvenis remetem para o hedonismo, o experimentalismo e o presentismo (Ferreira, 2011; Pais, 1990), que se constituem enquanto potenciadores de adesão aos consumos de performance, no sentido em que estes permitem alcançar, de forma mais rápida e com menos esforço, os objectivos que esses contextos suscitam.

Em terceiro lugar, o perfil de actividade dos jovens inquiridos (estudantes do Ensino Superior ou trabalhadores sem formação superior) veio a confirmar-se como uma variável significativamente diferenciadora das atitudes face aos consumos de performance. Em concreto, os estudantes do Ensino Superior manifestam maior preocupação com a performance neuro/cognitiva, ao passo que os trabalhadores revelam maior adesão atitudinal aos investimentos para a performance físico/corporal. O mesmo se pode afirmar a propósito do género, com as jovens a revelarem maior preocupação com a gestão do desempenho neuro/cognitivo, enquanto os jovens estão mais direccionados para investimentos com finalidades de performance física/corporal. Tais relações vêm renovar a necessidade de ter em consideração as teses que continuam a defender uma análise das culturas juvenis socialmente enraizada na estrutura social (Shildrick e MacDonald, 2006).

Em suma, os resultados são reveladores de uma relativa permeabilidade nas culturas juvenis à ideia de gestão da performance e mesmo ao desejo de melhoria do desempenho nas esferas neuro/cognitiva e físico/corporal. No entanto, esta permeabilidade não é homogénea e assume modulações, quer em função de critérios de legitimação dos consumos de performance, quer em termos da diversidade social que caracteriza a juventude e dos contextos em que os jovens se situam.

Finalmente, o retrato extensivo traçado permite identificar um conjunto de pistas analíticas merecedoras de exploração futura. Entre esses questionamentos destaca-se, em primeiro lugar, o aprofundamento dos contextos particulares em que os jovens se movem, incluindo, para além da segmentação imediata entre contexto académico e contexto laboral, a atenção a universos específicos, como sejam os ligados a determinados tipos de prática desportiva, na medida em que constituam nichos de disposições sociais para certos consumos de performance. Em segundo lugar, a exploração de culturas de performance juvenis, procurando perce- ber os sentidos atribuídos à performance, tanto ao nível físico/corporal como ao nível neuro/cognitivo, bem como os investimentos não terapeuticalizados (sem recurso a medicamentos ou outros produtos) que os jovens fazem com finalidades de manutenção ou melhoria do seu desempenho a esses níveis. Por fim, a compreensão das lógicas de legitimação dos consumos de performance e o modo como essas lógicas vão sendo construídas e alimentadas por práticas de consumo ou de não consumo, bem como por atitudes onde se cruzam percepções sobre o risco, concepções sobre o corpo, ou representações sobre o natural e o farmacológico.

\section{Agradecimentos}

Um agradecimento a todos os jovens - estudantes e trabalhadores - que participaram no estudo, bem como aos responsáveis nas instituições - universidades e empresas - que possibilitaram a recolha de informação.

\section{Notas}

1 O projecto "Consumos terapêuticos de performance na população jovem: trajectórias e redes de informação", financiado pela Fundação para a Ciência e Tecnologia (PTDC/CS-SOC/118073/2010), foi desenvolvido no quadro do Centro de Investigação e Estudos de Sociologia, do ISCTE-Instituto Universitário de Lisboa (CIES-IUL), em parceria com o Centro de Investigação Interdisciplinar Egas Moniz (CiiEM). Para além da autora do presente artigo, fizeram parte da equipa de investigação Noémia Lopes (coordenadora), Telmo Clamote, Hélder Raposo, Carla Rodrigues e Ana Isabel Fernandes.

2 Em 2014, 31,4\% dos jovens entre os 25 e os 34 anos tinham o Ensino Superior completo, enquanto a média da União Europeia era de 37,2\% (Eurostat: http://appsso. eurostat.ec.europa.eu/nui/submitViewTableAction.do).

3 No quadro da pesquisa, a categoria consumos de performance foi operacionalizada através da enunciação de um conjunto de medicamentos e produtos naturais para diferentes finalidades: 1) no domínio do desempenho neuro/cognitivo (para dormir, para a concentração, para descontrair/acalmar); 2) e no domínio do desempenho físico/corporal (para aumentar a energia física, para emagrecer, para aumentar a massa muscular) (cf. Relatório Estatístico do projecto, Lopes, 2014, pp. xiii, disponível em http://www.cies.iscte.pt/Consumos_de_Performance. pdf).

4 A amostra dos estudantes do Ensino Superior não inclui trabalhadores-estudantes.

5 Para uma reflexão sobre questões metodológicas no estudo de consumos terapêuticos ver Rodrigues, 2010.

6 Todos os dados apresentados e analisados ao longo do artigo, bem como o inquérito por questionário, podem ser consultados no Relatório Estatístico do projecto de investigação (Lopes, 2014), disponível em: http://www. cies.iscte.pt/Consumos_de_Performance.pdf.

7 Refira-se que 65,8\% dos jovens inquiridos já usaram algum tipo de fármaco ou produto natural para a 
gestão do desempenho. Para uma análise detalhada da expressão dos consumos de performance na população jovem, ver Lopes et al., 2015 e Lopes e Rodrigues, 2015.

8 Operacionalizado no questionário através da questão "Indique qual considera que seria a reacção dos(as) seus/suas amigos(a)s se soubessem que tomava algum dos seguintes medicamentos ou produtos naturais para melhorar o seu desempenho físico, intelectual ou social?" (cf. Lopes, 2014).

9 A introdução do haxixe e da marijuana na lista de produtos sobre os quais se procura captar percepções de legitimidade não corresponde a um entendimento de que estes produtos façam parte do quadro de consumos de performance considerados no estudo, mas teve como objectivo incluir uma referência de um consumo com alguma expressão entre a população jovem (ou com o qual está familiarizada), de forma a estabelecer comparações com as atitudes face aos consumos com finalidades de performance.

\section{Referências bibliográficas}

CLAMOTE, T. C. (2015), "Reverberações da medicalização: paisagens e trajetórias informacionais em consumos de performance", Sociologia, Revista da Faculdade de Letras da Universidade do Porto, vol. XXIX, pp. 35-57.

COSTA, A. F. e J. T. Lopes (orgs.) (2008), Os estudantes e os seus trajectos no Ensino Superior, CIES-IUL, IS UP (Relatório Final).

COVENEY, C.; J. Gabe e S. Williams (2011), "The Sociology of Cognitive Enhancement: Medicalisation and Beyond", Health Sociology Review, 20 (4), pp. 381-393.

EHRENBERG, A. (1991), Le culte de la performance, Paris, Calmant Lévy.

FEATHERSTONE, M. (1982), "The Body in Consumer Culture", Theory, Culture and Society, 1, pp. 18-33.

FERREIRA, V. (1986), "O inquérito por questionário na construção de dados sociológicos", in A. S. Silva e J. M. Pinto (eds.), Metodologia das Ciências Sociais, Porto, Afrontamento.

FERREIRA, V. S. (2003), "Atitudes dos jovens portugueses perante o corpo", in J. M. Pais e M. V. Cabral (eds.), Condutas de risco, práticas culturais e atitudes perante o corpo, Oeiras, Celta Editora, pp. 246-263.

FERREIRA, V. S. (2011), "Dar corpo à juventude: o corpo jovem e os jovens nos seus corpos", in J. M. Pais, R. Bendit, e V. S. Ferreira (eds.), Jovens e rumos, Lisboa, Imprensa de Ciências Sociais, pp. 257-275.

HEILBRUNN, B. (ed.) (2004), La performance, une nouvelle idéologie?, Paris, Éditions La Découverte.
LOPES, N. (org.) (2014), Relatório estatístico - Consumos terapêuticos de performance na população jovem, CIES-IUL. http://www.cies.iscte.pt/Consumos_de_Performance.pdf

LOPES, N. e C. Rodrigues (2015), "Medicamentos, consumos de performance e culturas terapêuticas em mudança", Sociologia, Problemas e Práticas, n. 078 , pp. 9-28.

LOPES, N. M. (org.) (2010), Medicamentos e pluralismo terapêutico: práticas e lógicas sociais em mudança, Porto, Afrontamento.

LOPES, N.; T. Clamote; H. Raposo; E. Pegado e C. Rodrigues (2012), "O natural e o farmacológico: padrões de consumo terapêutico na população portuguesa", Saúde \& Tecnologia, 8, pp. 5-17.

LOPES, N.; T. Clamote; H. Raposo; E. Pegado e C. Rodrigues (2015), "Medications, Youth Therapeutic Cultures and Performance Consumptions: A Sociological Approach", Health, 19 (4), pp. 430-448.

MOURA FERREIRA, P. (2003), "Comportamentos de risco dos jovens", in J. M. Pais e M. V. Cabral (eds.), Condutas de risco, práticas culturais e atitudes perante o corpo, Oeiras, Celta Editora, pp. 41-166.

PAIS, J. M. (1990), "A construção sociológica da juventude - Alguns contributos", Análise Social, 25 (105/106), pp. 139-165.

PAIS, J. M. (2000), "Transitions and Youth Cultures: Forms and Performances", International Social Science Journal, 52 (164), pp. 219-232.

PEGADO, E. (2010), "Consumos terapêuticos e investimentos de saúde", in N. M. Lopes (org.), Medicamentos e pluralismo terapêutico: práticas e lógicas sociais em mudança, Porto, Afrontamento, pp. 223-266.

RAPOSO, H. (2010), "Consumos terapêuticos, percepção e gestão do risco", in N. M. Lopes (org.), Medicamentos e pluralismo terapêutico: práticas e lógicas sociais em mudança, Porto, Afrontamento, pp. 159-222.

RAPOSO, H. (2016), "O risco e os consumos de performance na população jovem: entre as conceções e as práticas", Revista Portuguesa de Saúde Pública, 34 (2), pp. 186-195.

RODRIGUES, C. F. (2010), "Consumos terapêuticos: notas e reflexões metodológicas", in N. M. Lopes (org.), Medicamentos e pluralismo terapêutico: práticas e lógicas sociais em mudança, Porto, Afrontamento, pp. 267-282.

SHILDRICK, T. e R. MacDonald (2006), "In Defense of Subculture: Young People, Leisure and Social Divisions", Journal of Youth Studies, 9 (2), pp.125-140.

SHILLING, C. (1993), The Body and Social Theory, Londres, Sage Publications.

WILLIAMS. S. e S. Boden (2004), "Consumed with Sleep? Dormant Bodies in Consumer Culture", Sociological Research Online, 9 (2). (<http:// www.socresonline.org.uk/9/2/williams.html >)

Recebido a 24/04/2014. Aceite para publicação a 11/10/2016.

Elsa Pegado (elsa.pegado@iscte.pt). Instituto Universitário de Lisboa (ISCTE-IUL), Centro de Investigação e Estudos de Sociologia (CIES-IUL) \& Egas Moniz, Centro de Investigação Interdisciplinar (CiiEM). CIES-IUL, Edifício ISCTE, Av. das Forças Armadas, 1649-026 Lisboa, Portugal. 